\title{
Experimental design and performance analysis in plasma arc surface hardening
}

\begin{abstract}
In this paper, the experimental design by using the Taguchi method was employed to optimize the processing parameters in the plasma arc surface hardening process. The evaluated processing parameters are arc current, scanning velocity and carbon content of steel. In addition, the significant effects of the relation between processing parameters were also investigated. An orthogonal array, signal-to-noise $(\mathrm{S} / \mathrm{N})$ ratio and analysis of variance (ANOVA) were employed to investigate the effects of these processing parameters. Through this study, not only the increasing in hardened depth and improvement in surface roughness, but the parameters that significantly affect on the hardening performance were also identified. Experimental results showed the effectiveness of this approach.
\end{abstract}

Keyword: ANOVA; Hardened depth; Optimization; Plasma arc; Surface roughness; Taguchi method 